\title{
Glomerular Neovascularization in Nondiabetic Renal Allograft Is Associated with Calcineurin Inhibitor Toxicity
}

\author{
Anri Sawada ${ }^{a}$ b Masayoshi Okumi ${ }^{c}$ Shigeru Horita ${ }^{d}$ Kohei Unagamie Sekiko Taneda $^{f}$ \\ Shohei Fuchinoue ${ }^{g}$ Hideki Ishida $^{\mathrm{e}}$ Motoshi Hattori $^{\mathrm{h}}$ Kazunari Tanabe ${ }^{\mathrm{c}}$ Kosaku Nitta $^{\mathrm{i}}$ \\ Junki Koikej Yoji Nagashimab ${ }^{\mathrm{j}}$ Akira Shimizu $^{\mathrm{a}}$ \\ aDepartment of Analytic Human Pathology, Nippon Medical School, Tokyo, Japan; 'bepartment of Surgical \\ Pathology, Tokyo Women's Medical University, Tokyo, Japan; 'Department of Urology, Tokyo Women's Medical \\ University, Tokyo, Japan; 'Division of Clinical Pathology, Kidney Center, Tokyo Women's Medical University, Tokyo, \\ Japan; 'Department of Organ Transplant Medicine, Tokyo Women's Medical University, Tokyo, Japan; fDepartment \\ of Pathology, Tokyo Women's Medical University, Tokyo, Japan; ' ${ }^{D}$ epartment of Kidney Surgery, Tokyo Women's \\ Medical University, Tokyo, Japan; ' ${ }^{2}$ epartment of Pediatric Nephrology, Tokyo Women's Medical University, Tokyo, \\ Japan; 'Department of Nephrology, Tokyo Women's Medical University, Tokyo, Japan; 'Department of Pathology, St. \\ Marianna University School of Medicine, Kawasaki, Japan
}

\section{Keywords}

Calcineurin inhibitor · Diabetes mellitus · Glomerular neovascularization · Kidney transplantation · Polar vasculosis

\begin{abstract}
Introduction: Extra efferent arterioles, also known as polar vasculosis (PV), are often observed in the glomerular vascular pole and are associated with glomerular hypertrophy, indicating early recurrent diabetic kidney disease (DKD) in renal allografts. However, its significance in patients without diabetes remains uncertain. Methods: A total of 9,004 renal allograft biopsy specimens obtained between January 2007 and December 2017 at Tokyo Women's Medical University were retrospectively analyzed to examine the clinical and pathological significance of PV in renal allografts. PV was identified in 186 biopsy specimens obtained from 165 patients. The PV group comprised 46 patients; 35 patients without DKD and 11 patients with DKD as the initial cause of ESRD, whose clinical information was available and treated
\end{abstract}

with the calcineurin inhibitor (CNI) tacrolimus. The non-PV group comprising patients with renal allografts matched for age and postoperative day included 93 patients without DKD and 16 patients with DKD as the initial cause of ESRD. Results: In patients with nondiabetic renal allografts, systolic blood pressure was significantly higher in the PV group than in the non-PV group. The trough tacrolimus levels during the overall study period and at 2 weeks, 1 month, and 2 years after transplantation were significantly higher in the PV group compared with the non-PV group. Glomerulomegaly was significantly more common. Moreover, ah and aah scores in Banff score were significantly higher in the PV group than in the non-PV group. In those with diabetic renal allografts, although the clinical parameters and tacrolimus trough levels in all time periods were not significantly different between the PV and non-PV groups, the ah score was significantly higher in the PV group. Conclusion: PV was associated with CNI toxicity in nondiabetic but not in diabetic renal allografts. The pathogenesis of PV in renal allografts is considered to be multifactorial.

(c) 2020 S. Karger AG, Basel

$\begin{aligned} & \text { karger@karger.com } \\ & \text { www.karger.com/nef }\end{aligned}$
Karger ${ }^{\prime /}$

Anri Sawada

Department of Analytic Human Pathology, Nippon Medical School 1-1-5, Sendagi, Bunkyo-ku

Tokyo 113-8602 (Japan)

anri-sawada@nms.ac.jp 


\section{Introduction}

Diabetes leads to abnormal angiogenesis because of tissue ischemia and hyperglycemia [1]. New vessel formation in glomeruli has been reported in diabetic kidney disease (DKD), and one study previously demonstrated glomerular neovascularization in association with hypertrophy [2]. Moreover, extra efferent arterioles are often observed in the glomerular vascular pole; this morphological change is referred to as polar vasculosis (PV) and is considered a characteristic lesion of diabetic nephropathy [3]. In renal allografts, PV is an early sign of early recurrent DKD [4]. However, the significance of PV in nondiabetic renal allografts remains unclear. In the present study, we examined the clinical and pathological significance of PV in renal allografts of both diabetic and nondiabetic patients.

\section{Materials and Methods}

\section{Biopsy Sample Selection}

The present study included the analysis of 9,004 renal allograft biopsy specimens collected between January 2007 and December 2017 at Tokyo Women's Medical University. PV was detected in 186 biopsies collected from 165 patients, and clinical information was obtained from 48 patients. Two patients who were not treated by tacrolimus for immunosuppression were excluded. Therefore, the remaining 46 patients, including 35 patients without DKD and 11 patients with DKD as the initial cause of ESRD, were identified as the PV group. The non-PV group comprised patients with renal allografts matched for age and postoperative day and included 93 patients without DKD and 16 patients with DKD as the initial cause of ESRD. All patients of both PV and non-PV groups received standard maintenance immunosuppression therapy comprising steroids, tacrolimus, and antimetabolites. All patients underwent pretransplantation biopsies, and PV was not found in all patients with pretransplantation biopsies.

\section{Clinical Evaluation}

Patient information was reviewed using the medical records. All data were presented as means $\pm \mathrm{SD}$. The diagnostic criteria for diabetes by the Committee of Japan Diabetes Society were adopted in the present study [5].

\section{Histological Evaluation}

In our laboratory, all renal biopsies were examined using standard light microscopy (LM) and immunofluorescence. For LM, all biopsies were routinely stained with hematoxylin and eosin, periodic acid-Schiff, Masson trichrome, and periodic acid-methenamine silver. Pathological diagnosis was independently confirmed by 3 pathologists. Pathological scoring was performed based on the 2017 Banff classification [6]. Immunoglobulin A (IgA) nephropathy was diagnosed based on IgA deposition and complement 3 codeposition in mesangial lesions. Arteriosclerosis was categorized into mild, moderate, and severe stages according to the degree of internal elastic membrane involvement. Glomerulomegaly was defined as a glomerulus diameter $\geq 250 \mu \mathrm{m}$.

\section{Statistical Analysis}

Continuous variables were compared using the Mann-Whitney test, and categorical variables were compared using Fisher's exact test. Mean values of clinical parameters, including hemoglobin A1c ( $\mathrm{HbA1c})$, fasting plasma glucose, systolic blood pressure, and BMI, during the clinical course after renal transplantation were determined. All statistical analyses were performed using the JMP statistical software (SAS Institute, Cary, NC, USA). All $p$ values were two-sided, and a $p$ value $<0.05$ was considered to indicate statistical significance.

\section{Results}

\section{Clinical Characteristics of Patients without DKD}

We first examined the nondiabetic renal allografts. The clinical characteristics of patients in the PV and non-PV groups are summarized in Table 1. Briefly, among the clinical parameters, systolic blood pressure was significantly higher in the PV group than in the non-PV group $(p<0.05)$ whereas there were no significant differences in fasting plasma glucose, $\mathrm{HbA1c}$, and number of patients with posttransplant diabetes mellitus between the PV and non-PV groups.

\section{Tacrolimus Trough Levels during the Clinical Course} of Patients without DKD

The tacrolimus trough levels in specific periods after transplantation are presented in Table 2. Briefly, the trough levels of tacrolimus were significantly higher during the overall study period as well as at 2 weeks, 1 month, and 2 years after transplantation in the PV group than in the non-PV group ( $p<0.05$, respectively).

\section{Pathological Characteristics of Patients without DKD}

The pathological characteristics of patients without $\mathrm{DKD}$ in the PV and non-PV groups are provided in Table 3. Among the pathological findings, glomerulomegaly was significantly more common in patients without DKD in the PV group, and the ah score and the aah score were significantly higher in the PV group $(p<0.05$, respectively). These phenomena are sometimes observed in the same glomerulus. As shown in Figure 1a, the glomeruli exhibited moderate mesangial expansion with severe $\mathrm{PV}$, accompanied with arteriolar hyalinosis in the medial smooth muscles. Figure $1 \mathrm{~b}$ shows enlarged glomeruli with PV and ah in the media. In contrast, no significant correlations were noted in the number of the patients with FSGS lesion, mm score, ci score, glomerular obsolescence, and arteriosclerosis score. The number of patients with rejection (antibody-mediated rejection and T cell-mediated rejection) and IgA nephropathy was not significantly different.
Sawada et al. 
Table 1. Clinical characteristics of non-DKD patients

\begin{tabular}{lccc}
\hline & With PV, $n=35$ & Without PV, $n=93$ & $p$ value \\
\hline POD & $1,850.1 \pm 2,235.7$ & $1,924.8 \pm 1,997.6$ & 0.12 \\
Age, years & $45.1 \pm 13.1$ & $47.0 \pm 12.4$ & 0.36 \\
Sex (male) & 26 & 53 & 0.10 \\
Donor age, years & $60.9 \pm 8.6$ & $58.1 \pm 9.7$ & 0.16 \\
Donor sex (male) & 18 & 33 & 0.10 \\
Unrelated donor & 2 & 2 & 0.30 \\
ABO incompatibility & 15 & 40 & 0.83 \\
Cause of ESRD (IgA nephropathy) & 6 & 27 & 0.26 \\
Duration of dialysis, months & $42.5 \pm 51.5$ & $51.6 \pm 83.7$ & 0.34 \\
HLA-AB mismatches & $1.6 \pm 1.0$ & $1.9 \pm 1.0$ & 0.92 \\
HLA-DR mismatches & $0.7 \pm 0.6$ & $1.0 \pm 0.6$ & 0.71 \\
HbAlc, $\%$ & $5.3 \pm 0.3$ & $5.5 \pm 0.7$ & 0.11 \\
Fasting plasma glucose, mg/dL & $100.6 \pm 9.2$ & $106.2 \pm 23.4$ & 0.10 \\
PTDM & 1 & 6 & 0.67 \\
Systolic blood pressure, mm Hg & $130.4 \pm 12.2$ & $125.8 \pm 10.7$ & $<0.05$ \\
BMI, kg/m & $21.5 \pm 2.8$ & $20.8 \pm 3.0$ & 0.18 \\
Graft loss & 1 & 5 & 1.00 \\
\hline
\end{tabular}

Data are presented as mean \pm SD. Categorical variables were compared by Fisher's exact test, and continuous variables were compared by the Mann-Whitney test. DKD, diabetic kidney disease; HLA, human leukocyte antigen; HbA1c, hemoglobin A1c; PTDM, posttransplant diabetic mellitus; SD, standard deviation; PV, polar vasculosis.

Table 2. Tacrolimus trough level in the clinical course of non-DKD patients

\begin{tabular}{lrlr}
\hline $\begin{array}{l}\text { FK trough, } \\
\text { ng/mL }\end{array}$ & $\begin{array}{l}\text { With PV, } \\
n=35\end{array}$ & $\begin{array}{l}\text { Without PV, } \\
n=93\end{array}$ & $\begin{array}{l}p \\
\text { value }\end{array}$ \\
\hline 2 weeks & $10.2 \pm 6.1$ & $7.8 \pm 3.2$ & $<0.05$ \\
1 month & $8.4 \pm 3.9$ & $7.1 \pm 2.0$ & $<0.05$ \\
3 months & $7.0 \pm 2.7$ & $6.4 \pm 1.8$ & 0.07 \\
6 months & $6.0 \pm 2.1$ & $5.8 \pm 1.6$ & 0.11 \\
1 year & $5.1 \pm 1.3$ & $5.4 \pm 1.3$ & 0.11 \\
2 years & $5.4 \pm 1.3$ & $4.9 \pm 1.4$ & $<0.05$ \\
3 years & $4.5 \pm 0.9$ & $4.5 \pm 1.3$ & 0.12 \\
4 years & $5.2 \pm 1.6$ & $4.8 \pm 1.1$ & 0.08 \\
Mean & $6.6 \pm 1.3$ & $5.8 \pm 0.8$ & $<0.05$ \\
\hline
\end{tabular}

Data are presented as mean \pm SD. Categorical variables were compared by Fisher's exact test, and continuous variables were compared by the Mann-Whitney test. DKD, diabetic kidney disease; CyA, cyclosporine; FK, tacrolimus; PV, polar vasculosis; SD, standard deviation.

Comparison of the Clinical and Pathological Findings of Patients with DKD as the Initial Cause of ESRD between the PV and Non-PV Groups

We next compared the clinical and pathological findings of patients with DKD as the initial cause of ESRD
Table 3. Pathological findings of non-DKD patients

\begin{tabular}{lccc}
\hline & $\begin{array}{l}\text { With PV, } \\
n=35\end{array}$ & $\begin{array}{l}\text { Without PV, } \\
n=93\end{array}$ & \\
& & 3 & $<0.05$ \\
Glomerulomegaly $(\geq 250 \mu \mathrm{m})$ & 8 & 3 & \\
Glomerular obsolescence, $\%$ & $16.0 \pm 18.0$ & $18.1 \pm 18.5$ & 0.61 \\
FSGS lesion & 4 & 4 & 0.21 \\
mm score (score: $0-3)$ & $0.18 \pm 0.52$ & $0.032 \pm 0.18$ & 0.059 \\
ci score (score: $0-3)$ & $0.59 \pm 0.70$ & $0.39 \pm 0.68$ & 0.075 \\
ah score (score: $0-3)$ & $1.5 \pm 1.0$ & $0.94 \pm 1.0$ & $<0.05$ \\
aah score (score: $0-3)$ & $0.97 \pm 1.1$ & $0.47 \pm 0.80$ & $<0.05$ \\
Arteriosclerosis (score: $0-3)$ & $1.3 \pm 0.94$ & $1.2 \pm 0.87$ & 0.77 \\
IgA nephropathy & 2 & 5 & 1.0 \\
Antibody-mediated rejection & 3 & 17 & 0.27 \\
T cell-mediated rejection & 0 & 4 & 0.57
\end{tabular}

Data are presented as mean $\pm \mathrm{SD}$. Categorical variables were compared by Fisher's exact test, and continuous variables were compared by the Mann-Whitney test. DKD, diabetic kidney disease; FSGS, focal segmental glomerulosclerosis; PV, polar vasculosis; SD, standard deviation; POD, postoperative day.

between the PV and non-PV groups. The clinical characteristics and the tacrolimus trough levels in specific periods after transplantation are provided in Tables 4 and 5 . There were no significant differences in none of the clinical findings and tacrolimus trough levels between the 2 
Table 4. Clinical characteristics of DKD patients

\begin{tabular}{lccl}
\hline & With PV, & Without PV, & $p$ \\
& $n=11$ & $n=16$ & value \\
\hline POD & $1,512.7 \pm 1,560.9$ & $1,159.9 \pm 929.1$ & 0.96 \\
Age, years & $63.2 \pm 8.9$ & $58.9 \pm 9.3$ & 0.16 \\
Sex (male) & 9 & 12 & 1.0 \\
Donor age, years & $59.7 \pm 5.1$ & $58.8 \pm 8.6$ & 0.77 \\
Donor sex (male) & 1 & 6 & 0.18 \\
Unrelated donor & 0 & 0 & 1.0 \\
ABO incompatibility & 9 & 10 & 0.40 \\
Duration of dialysis, months & $32.7 \pm 36.3$ & $26.4 \pm 27.6$ & 0.60 \\
HLA-AB mismatches & $3.0 \pm 0.6$ & $2.4 \pm 1.1$ & 0.16 \\
HLA-DR mismatches & $1.7 \pm 0.47$ & $1.3 \pm 0.68$ & 0.060 \\
HbA1c, \% & $6.5 \pm 0.52$ & $6.4 \pm 0.74$ & 0.96 \\
Fasting plasma glucose, mg/dL & $100.6 \pm 9.2$ & $106.2 \pm 23.4$ & 0.10 \\
PTDM & 7 & 11 & 1.0 \\
Systolic blood pressure, mm Hg & $133.0 \pm 13.4$ & $130.0 \pm 11.2$ & 0.55 \\
BMI, kg/m ${ }^{2}$ & $23.0 \pm 2.7$ & $23.1 \pm 3.1$ & 0.69 \\
Graft loss & 1 & 1 & 1.00 \\
\hline
\end{tabular}

Data are presented as mean $\pm \mathrm{SD}$. Categorical variables were compared by Fisher's exact test, and continuous variables were compared by the Mann-Whitney test. DKD, diabetic kidney disease; HLA, human leukocyte antigen; Hb, hemoglobin; SD, standard deviation; PTDM, posttransplant diabetes mellitus; PV, polar vasculosis; POD, postoperative day.

Table 5. Tacrolimus trough level in the clinical course of DKD patients

\begin{tabular}{llll}
\hline $\begin{array}{l}\text { FK trough, } \\
\text { ng/mL }\end{array}$ & $\begin{array}{l}\text { With PV, } \\
n=11\end{array}$ & $\begin{array}{l}\text { Without PV, } \\
n=16\end{array}$ & $\begin{array}{l}p \\
\text { value }\end{array}$ \\
\hline 2 weeks & $9.5 \pm 5.4$ & $7.6 \pm 2.0$ & 0.17 \\
1 month & $7.9 \pm 5.1$ & $6.5 \pm 1.4$ & 0.80 \\
3 months & $6.1 \pm 1.6$ & $5.9 \pm 1.1$ & 0.98 \\
6 months & $5.9 \pm 1.5$ & $5.2 \pm 1.2$ & 0.36 \\
1 year & $5.5 \pm 1.3$ & $5.3 \pm 1.3$ & 0.91 \\
2 years & $4.6 \pm 1.2$ & $4.4 \pm 1.1$ & 0.59 \\
3 years & $4.0 \pm 0.91$ & $4.8 \pm 1.2$ & 0.20 \\
4 years & $4.4 \pm 1.1$ & $4.4 \pm 1.0$ & 1.0 \\
Mean & $6.1 \pm 1.1$ & $5.9 \pm 1.1$ & 0.52 \\
\hline
\end{tabular}

Data are presented as mean $\pm \mathrm{SD}$. Categorical variables were compared by Fisher's exact test, and continuous variables were compared by the Mann-Whitney test. DKD, diabetic kidney disease; CyA, cyclosporine; FK, tacrolimus; PV, polar vasculosis; $\mathrm{SD}$, standard deviation.

groups. The pathological characteristics of patients with DKD are presented in Table 6. Among the pathological findings, the ah score was significantly higher in the PV group than in the non-PV group. Albeit not significant,

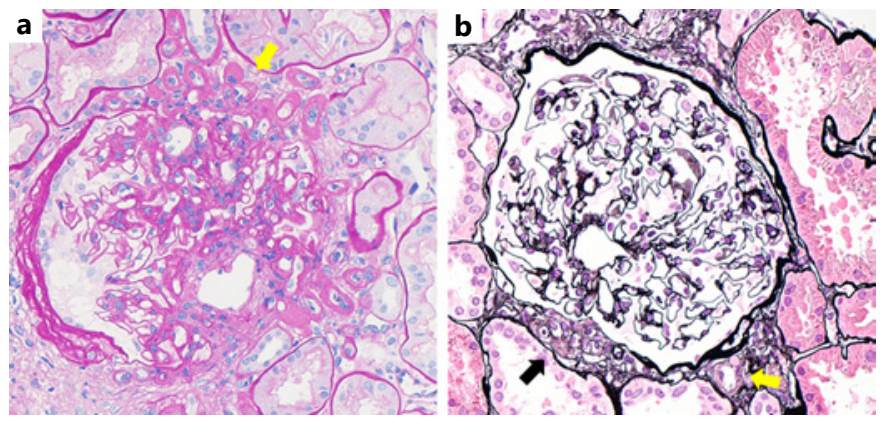

Fig. 1. Characteristic histological findings of kidney biopsy specimens obtained from patients without diabetic kidney disease: a allograft biopsy was performed in postoperative day (POD) 2500. Glomeruli show moderate mesangial expansion with severe PV, accompanied with ah in media (yellow arrow) (i0, t0, ti0, i-IFTA0, v0, g0, ptc0, ci1, ct1, cv0, ti0, mm2, cg0, ptcbm0, ah3, aah3, and $\mathrm{C} 4 \mathrm{~d} 0$ according to the Banff classification). Immunoglobulin and complement depositions are not found in glomeruli. Initial cause of ESRD of this patient is not diabetic nephropathy. This patient did not develop PTDM. b Allograft biopsy was performed in POD 9984. Note enlarged glomeruli with PV (black arrow) and ah in media (yellow arrow) (i0, t0, ti0, i-IFTA0, v0, g0, ptc0, ci1, ct1, cv0, ti0, mm0, cg0, ptcbm0, ah2, aah1, and C4d0 according to the Banff classification). Immunoglobulin and complement depositions are not observed in glomeruli. Initial cause of ESRD of this patient is not diabetic nephropathy. This patient did not develop PTDM. Periodic acid-Schiff, $\times 20$ (a); periodic acid-methenamine-silver, $\times 20$ (b).

there was a tendency for more glomerular hypertrophy in the PV group than in the non-PV group. No specific trends were found for other pathological features.

\section{Discussion}

$\mathrm{PV}$, a characteristic lesion of diabetic nephropathy, is considered a part of abnormal angiogenesis due to diabetes. However, PV has also been observed in patients other than those with DKD including those with renal allografts, and no clinicopathological study to date has focused on PV. Our analyses suggested that PV was associated with calcineurin inhibitor (CNI) toxicity in nondiabetic renal allografts.

$\mathrm{CNI}$ is an indispensable drug that acts as an immunosuppressant after transplantation. CNI is frequently associated with various side effects such as hypertension, hyperkalemia, metabolic acidosis, and hypercalciuria [7]. Depending on the length of posttransplantation period, CNI causes various pathological changes in renal allografts. In the early phase after transplantation, CNI tox-
40
Nephron 2020;144(suppl 1):37-42 DOI: $10.1159 / 000511452$
Sawada et al. 
Table 6. Pathological findings of DKD patients

\begin{tabular}{lccl}
\hline & $\begin{array}{l}\text { With PV, } \\
n=11\end{array}$ & $\begin{array}{l}\text { Without PV, } \\
n=16\end{array}$ & \\
& 3 & 0 & 0.056 \\
Glomerulomegaly, $\geq 250 \mu \mathrm{m}$ & 3 & 0.74 \\
Glomerular obsolescence, $\%$ & $20.0 \pm 19.5$ & $18.1 \pm 19.4$ & 0.74 \\
FSGS lesion & 0 & 0 & 1.0 \\
mm score (score: $0-3)$ & $0.27 \pm 0.47$ & $0.13 \pm 0.34$ & 0.37 \\
ci score (score: $0-3)$ & $0.27 \pm 0.47$ & $0.44 \pm 0.16$ & 1.0 \\
ah score (score: $0-3)$ & $1.5 \pm 0.69$ & $0.75 \pm 0.86$ & $<0.05$ \\
aah score (score: $0-3)$ & $0.91 \pm 1.0$ & $0.25 \pm 0.14$ & 0.21 \\
Arteriosclerosis (score: $0-3)$ & $1.2 \pm 0.87$ & $1.1 \pm 0.81$ & 0.89 \\
IgA nephropathy & 0 & 0 & 1.0 \\
Antibody-mediated rejection & 1 & 3 & 0.62 \\
T-cell-mediated rejection & 0 & 1 & 1.0 \\
\hline
\end{tabular}

Data are presented as mean \pm SD. Categorical variables were compared by Fisher's exact test, and continuous variables were compared by the Mann-Whitney test. DKD, diabetic kidney disease; FSGS, focal segmental glomerulosclerosis; PV, polar vasculosis; SD, standard deviation; Ig, Immunoglobulin.

icity causes tubular damage and arteriolar hyalinosis in patients with high trough CNI levels $[8,9]$. In the late phase after transplantation, CNI toxicity causes severe arteriolar hyalinosis and progressive glomerulosclerosis [10]. Arteriolar hyalinosis is a common side effect in both early and late phases after transplantation. Several previous studies have demonstrated a significant correlation between the degree of arteriolar hyalinosis and CNI dosage and exposure duration $[8,11]$. Arteriolar hyalinosis caused by $\mathrm{CNI}$ involves deposition of hyaline in media; this lesion can be distinguished from hyaline deposition in vascular subendothelium caused by hypertension and diabetes. Therefore, arteriolar hyalinosis caused by CNI (aah score) is distinguished from arteriolar hyalinosis (ah score) caused by other factors in the diagnosis of renal allograft biopsies [12]. In the present study, the tacrolimus trough level and aah score were significantly higher in the $\mathrm{PV}$ group than in the non-PV group in nondiabetic renal allografts. Although the mechanism of CNI-induced arteriolar hyalinosis is not yet fully known, a previous animal study has shown that endothelial cell transforming growth factor (TGF)- $\beta$ receptor activation causes renal arteriolar hyalinosis [13]. Other studies have reported aberrant neovascularization caused by diseases such as cancer and atherosclerosis as the consequence of inappropriate TGF- $\beta$ mediated angiogenic signaling [14]. In addition, treatment with CNI induces vascular endothelial growth factor (VEGF) expression through the activation of protein ki-

Polar Vasculosis in Renal Allograft nase $C$ and protein kinase $\delta$ [15], and VEGF stimulates angiogenesis and vascularization. A previous study indicated that increased VEGF production in renal collecting ducts results in disproportional neovascularization, worsening local hypoxia, and interstitial fibrosis [16]. These studies suggest that elevation of TGF- $\beta$ and VEGF due to CNI can lead to both aberrant neovascularization and arteriolar hyalinosis. In the present study, the ah and aah scores and glomerulomegaly were significantly higher in the PV group compared with the non-PV group in nondiabetic renal allografts, suggesting that PV is associated with glomerular hyperfiltration and CNI toxicity.

We also compared the clinical and pathological characteristics of patients with DKD as the initial cause of ESRD. In those with diabetic renal allografts, there was no significant difference in tacrolimus trough levels between the PV and non-PV groups. In contrast, there was the tendency for a higher ah score and more glomerular hypertrophy in the PV group. DKD is a multifactorial condition, in which several factors such as hyperglycemia, hypertension, lipid abnormalities, smoking, and albuminuria are entangled in a complicated web [17]. In the progression of disease, metabolic pathways leading to glucotoxicity, hemodynamic pathway (glomerular hypertension), and inflammation are considered important [18]. Our results indicated that PV was associated with ah score and glomerular hypertrophy, that is, with arteriosclerosis and hyperfiltration but not with CNI toxicity in patients with DKD. Previous studies of native kidney biopsies reported that PV was not only a common finding but was also associated with renal outcomes in patients with type 2 diabetes $[19,20]$. Taken together, these findings affirm that management of intraglomerular pressure by inhibiting renin-angiotensin-aldosterone, blood pressure, and blood glucose level is necessary for the prevention of DKD progression.

We acknowledge several limitations in the present study. First, this was a retrospective study. Thus, we could not obtain data on some of the laboratory parameters relevant to vascular lesions. Based on previous studies, PV might be associated with microalbuminuria. However, microalbuminuria was not evaluated in the outpatient clinic where the patients were followed. The association between PV and microalbuminuria thus warrants further investigation. Second, although evaluation by electron microscopy is necessary to diagnose initial pathological changes in DKD, the biopsies of patients without DKD were not evaluated by electron microscopy. Therefore, the potential contribution of diabetes could not be completely excluded. In conclusion, the 
present study findings suggest that $\mathrm{PV}$ is associated with $\mathrm{CNI}$ toxicity in nondiabetic but not in diabetic renal allografts.

\section{Acknowledgements}

We thank Hideki Nakayama and Mayuko Ohno for their technical support. The authors would like to thank Enago (http://www. enago.jp) for the English language review.

\section{Statement of Ethics}

This study was performed in compliance with the Declaration of Helsinki. Informed consent was obtained by optout as this was a retrospective study. This study and all its protocols were approved by the Ethics Committee at Tokyo Women's Medical University (No. 4840).

\section{Conflict of Interest Statement}

The authors have no conflicts of interest to declare.

\section{Funding Sources}

This study did not receive any funding.

\section{Author Contributions}

Conceptualization, J.K. and A.S.; methodology, A.S. and S.H.; formal analysis, A.S.; investigation, A.S., S.T., and, J.K.; resources, M.O., K.U., S.F., H.I., M.H., K.T., Y.N., and K.N.; writing - original draft, A.S.; review and editing, S.T., J.K.; supervision, A. Shimizu., Y.N., and K.N.; and project administration, Y.N. and K.N.

\section{References}

1 Nakagawa T, Kosugi T, Haneda M, Rivard CJ, Long DA. Abnormal angiogenesis in diabetic nephropathy. Diabetes. 2009 Jul;58(7):14718.

2 Osterby R, Asplund J, Bangstad HJ, Nyberg G, Rudberg S, Viberti GC, et al. Neovascularization at the vascular pole region in diabetic glomerulopathy. Nephrol Dial Transplant. 1999 Feb;14(2):348-52.

3 Min W, Yamanaka N. Three-dimensional analysis of increased vasculature around the glomerular vascular pole in diabetic nephropathy. Virchows Arch A Pathol Anat Histopathol. 1993;423(3):201-7.

4 Nyumura I, Honda K, Tanabe K, Teraoka S, Iwamoto Y. Early histologic lesions and risk factors for recurrence of diabetic kidney disease after kidney transplantation. Transplantation. 2012 Sep 27;94(6):612-9.

5 Seino Y, Seino Y, Nanjo K, Tajima N, Kadowaki T, Kashiwagi A, et al. Report of the committee on the classification and diagnostic criteria of diabetes mellitus. J Diabetes Investig. 2010 Oct 19;1(5):212-28.

6 Haas M, Loupy A, Lefaucheur C, Roufosse C, Glotz D, Seron D, et al. The Banff 2017 kidney meeting report: revised diagnostic criteria for chronic active $\mathrm{T}$ cell-mediated rejection, antibody-mediated rejection, and prospects for integrative endpoints for next-generation clinical trials. Am J Transplant. 2018 Feb; 18(2):293-307.

7 Hoorn EJ, Walsh SB, McCormick JA, Fürstenberg A, Yang CL, Roeschel T, et al. The calcineurin inhibitor tacrolimus activates the renal sodium chloride cotransporter to cause hypertension. Nat Med. 2011 Oct 2;17(10): 1304-9.

8 Nankivell BJ, Borrows RJ, Fung CL, O'Connell PJ, Chapman JR, Allen RD. Calcineurin inhibitor nephrotoxicity: longitudinal assessment by protocol histology. Transplantation. 2004 Aug 27;78(4):557-65.

9 Charney DA, Bhaskaran M, Molmenti E. Calcineurin inhibitor toxicity in a renal transplant recipient. NDT Plus. 2009 Apr;2(2):175-6.

10 Okamoto M, Akioka K, Ushigome H, Higuchi A, Nobori S, Ogino S, et al. Ten-year protocol biopsy findings of renal allografts in the calcineurin inhibitor era. Clin Transplant. 2006 Jul;20(Suppl 15): 16-9.

11 Falkenhain ME, Cosio FG, Sedmak DD. Progressive histologic injury in kidneys from heart and liver transplant recipients receiving cyclosporine. Transplantation. 1996 Aug; 62(3):364-70

12 Loupy A, Haas M, Solez K, Racusen L, Glotz D, Seron D, et al. The Banff 2015 kidney meeting report: current challenges in rejection classification and prospects for adopting molecular pathology. Am J Transplant. 2017 Jan; 17(1):28-41.

13 Chiasson VL, Jones KA, Kopriva SE, Mahajan A, Young KJ, Mitchell BM. Endothelial cell transforming growth factor- $\beta$ receptor activation causes tacrolimus-induced renal arteriolar hyalinosis. Kidney Int. 2012 Oct;82(8): 857-66.

14 Wang X, Abraham S, McKenzie JAG, Jeffs N, Swire M, Tripathi VB, et al. LRG1 promotes angiogenesis by modulating endothelial TGF- $\beta$ signalling. Nature. 2013 Jul 18; 499(7458):306-11.

15 Basu A, Banerjee P, Contreras AG, Flynn E, $\mathrm{Pal}$ S. Calcineurin inhibitor-induced and rasmediated overexpression of VEGF in renal cancer cells involves mTOR through the regulation of PRAS40. PLoS One. 2011 Aug;6(8): e23919.

16 Prokai A, Csohany R, Sziksz E, Pap D, Balicza-Himer L, Boros S, et al. Calcineurininhibition results in upregulation of local renin and subsequent vascular endothelial growth factor production in renal collecting ducts. Transplantation. 2016 Feb;100(2): 325-33.

17 KDOQI clinical practice guidelines and clinical practice recommendations for diabetes and chronic kidney disease. Am J Kidney Dis. 2007 Feb;49(2 Suppl 2):S12-154.

18 Tuttle KR, Bakris GL, Bilous RW, Chiang JL, de Boer IH, Goldstein-Fuchs J, et al. Diabetic kidney disease: a report from an ADA consensus conference. Diabetes Care. 2014 Oct; 37(10):2864-83.

19 Hoshino J, Furuichi K, Yamanouchi M, Mise K, Sekine A, Kawada M, et al. A new pathological scoring system by the Japanese classification to predict renal outcome in diabetic nephropathy. PloS One. 2018;13(2):e0190923.

20 Furuichi K, Yuzawa Y, Shimizu M, Hara A, Toyama T, Kitamura $\mathrm{H}$, et al. Nationwide multicentre kidney biopsy study of Japanese patients with type 2 diabetes. Nephrol Dial Transplant. 2018 Jan 1;33(1):138-48. 